\title{
In vivo breast cancer characterization imaging using two monoclonal antibodies activatably labeled with near infrared fluorophores
}

\author{
Kohei Sano, Makoto Mitsunaga, Takahito Nakajima, Peter L Choyke and Hisataka Kobayashi*
}

\begin{abstract}
Introduction: The gene expression profiles of cancer cells are closely related to their aggressiveness and metastatic potential. Antibody-based immunohistochemistry $(\mathrm{IHC})$ of tissue specimens is a common method of identifying expressed proteins in cancer cells and increasingly inform treatment decisions. Molecular imaging is a potential method of performing similar $\mathrm{IHC}$ studies in vivo without the requirement for biopsy or tumor excision. To date, antibody-based imaging has been limited by high background levels related to slow clearance, making such imaging practical. However, optically activatable imaging agents, which are only fluorescent when bound to their cognate receptor, open the possibility of doing in vivo multi-color IHC.

Methods: We describe the use of activatable, near infrared fluorescence-labeled AlexaFluor680 (Alexa680) conjugated panitumumab (Pan) targeted against human epidermal growth factor receptor (EGFR) (Pan-Alexa680) and Indocyanine Green (ICG) conjugated trastuzumab (Tra) targeted against human epidermal growth factor receptor type 2 (HER2) (Tra-ICG) were synthesized and evaluated in cells in vitro and in an orthotopic breast cancer mouse model in vivo.

Results: Pan-Alexa680 (self-quenched; SQ) and Tra-ICG were initially quenched but demonstrated a 5.2- and 50fold dequenching capacity under detergent treatment, respectively. In vitro microscopy and flow cytometry using MDA-MB-468 (EGFR+/HER2-) and 3T3/HER2 cells (EGFR-/HER2+), demonstrated specific fluorescence signal for each cell type based on binding to Pan-Alexa680(SQ) or Tra-ICG. An in vivo imaging study employing a cocktail of PanAlexa680(SQ) and Tra-ICG (each $50 \mu \mathrm{g}$ ) was injected into mice with orthotopic MDA-MB-468 and 3T3/HER2 tumors in the breast. Each probe visualized only the target-specific breast tumor.
\end{abstract}

Conclusions: Multi-color target-specific fluorescence breast cancer imaging can be achieved in vivo by employing two activatable fluorescent probes administered as a cocktail. The images allowed us to see a specific receptor expression in each breast tumor without post-image processing.

\section{Introduction}

Antibodies are macromolecules with high binding specificity for target antigens. This makes them excellent scaffolds for molecular imaging probes. However, a central limitation of using antibodies for imaging is that they are slow to clear from the body resulting in nonspecific background contamination, reducing the targetto-background ratio. In order to overcome the high background signal found with labeled intact antibodies,

\footnotetext{
* Correspondence: kobayash@mail.nih.gov

Molecular Imaging Program, Center for Cancer Research, National Cancer Institute, NIH, Building 10, RoomB3B69, 10 Center Dr. Bethesda, MD 208921088, USA
}

(c) 2012 Sano et al.; licensee BioMed Central Ltd. This is an open access article distributed under the terms of the Creative Commons Attribution License (http://creativecommons.org/licenses/by/2.0), which permits unrestricted use, distribution, and reproduction in any medium, provided the original work is properly cited. ance from the blood pool leads to lower concentrations and, therefore, lower tumor uptake [1].

Optical imaging has many theoretical advantages for tumor identification and characterization. A central advantage is that simultaneous multi-color imaging can be employed to characterize expression profiles $[2,3]$. By using two or more different antibodies labeled with distinct colors, unbound antibody in the normal tissue can be mathematically subtracted $[4,5]$, since the bio-distribution of antibodies is similar in normal organs and only differs markedly at the tissue expressing the target antigen. clearance, have been developed. However, faster clear-

labeled antibody fragments, which show more rapid 
However, this approach is somewhat inefficient as it subtracts the target signal as well as the background signal. Therefore, another approach to reducing background signal from two-antibody-based optical is to take advantage of the unique feature of fluorescence that allows it to be quenched or activated depending on its chemical state. Such activatable probes (for example, labeled antibodies) are designed to generate signal only after being bound and processed by the target cancer cells; therefore, high accumulation of antibodies in the target tissue can be clearly visualized while the background signal remains quenched $[6,7]$. This approach permits many high tumor-to-background ratios to be achieved [8].

In this study, two different antibodies against human epidermal growth factor receptor (EGFR) and human epidermal growth factor receptor type 2 (HER2) were conjugated with activatable near-infrared (NIR) fluorophores, namely Indocyanine Green (ICG) and AlexaFluor680 (Alexa680), which emit light at different wavelengths. We then employed these two activatable antibodies as a cocktail in mice with EGFR and HER2 positive tumors in order to demonstrate the feasibility of this approach.

\section{Materials and methods Reagents}

Panitumumab (Pan), a fully human $\operatorname{IgG}_{2}$ monoclonal antibody (mAb) directed against the extracellular domain of the human EGFR (HER1), was purchased from Amgen (Thousand Oaks, CA, USA). Trastuzumab (Tra), a recombinant humanized $\mathrm{mAb}$ directed against the human HER2, was purchased from Genentech Inc. (South San Francisco, CA, USA). ICG-Sulfo-OSu was purchased from Dojindo Molecular Technologies (Rockville, MD, USA). Alexa680-NHS ester was purchased from Invitrogen Co. (Carlsbad, CA, USA). All other chemicals used were of reagent grade.

\section{Synthesis of Alexa680 or ICG conjugated antibodies}

Panitumumab $(0.5 \mathrm{mg}, 3.4 \mathrm{nmol})$ was incubated with Alexa680-NHS ester $(39.3 \mu \mathrm{g}, 34 \mathrm{nmol})$ in $0.1 \mathrm{M}$ $\mathrm{Na}_{2} \mathrm{HPO}_{4}(\mathrm{pH} 8.6)$ at room temperature for one hour, followed by the purification with a size exclusion column (PD-10; GE Healthcare, Piscataway, NJ, USA). ICG labeling of trastuzumab was also performed by reacting $\mathrm{mAb}$ with ICG at a ratio of 1:6 in the same manner as PanAlexa680. The concentrations of Alexa680 and ICG were calculated by measuring the absorption with the UV-Vis system (8453 Value UV-Vis system; Agilent Technologies, Santa Clara, CA, USA) to confirm the number of fluorophore molecules conjugated with each antibody molecule. The protein concentration was also determined by measuring the absorption at $280 \mathrm{~nm}$ with a UV-Vis system. The number of Alexa680 and ICG per antibody was adjusted to approximately 4.0 to 4.5 and 0.7 to 1.0 , respectively. As a comparison, Pan conjugated with approximately one Alexa680 (always-on type; PanAlexa680 (ON)) was also synthesized.

\section{Determination of quenching capacity in vitro}

The quenching abilities of each conjugate were investigated by denaturing them with $1 \%$ SDS as described previously [9]. Briefly, the conjugates were incubated with $1 \%$ sodium dodecyl sulfate (SDS) in PBS for 15 minutes at room temperature. As a control, the samples were incubated in PBS. The fluorescence signal intensity of Pan-Alexa680 was measured with a fluorescence spectrometer (Perkin-Elmer LS55, Perkin-Elmer, Shelton, CT, USA). The change in fluorescence intensity of ICG was investigated with an in vivo imaging system (Maestro, CRi Inc., Woburn, MA, USA) using 710 to $760 \mathrm{~nm}$ excitation and $800 \mathrm{~nm}$ long-pass emission filters.

\section{Cell culture}

The EGFR+/HER2- breast cancer cell line, MDA-MB-468, and the EGFR-/HER2+ cell line, NIH/3T3 (3T3/HER2), were used. Both cell lines were grown in RPMI 1640 (Life Technologies, Gaithersburg, MD, USA) containing 10\% fetal bovine serum (Life Technologies), 0.03\% L-glutamine, 100 units $/ \mathrm{mL}$ penicillin, and $100 \mu \mathrm{g} / \mathrm{mL}$ streptomycin in $5 \% \mathrm{CO}_{2}$ at $37^{\circ} \mathrm{C}$.

\section{Fluorescence microscopy studies}

MDA-MB-468 cells or 3T3/HER2 cells $\left(1 \times 10^{4}\right)$ were plated on a covered glass-bottomed culture well and incubated for 16 hours. Pan-Alexa680 (ON or self-quenched; $\mathrm{SQ})$ and Tra-ICG $(10 \mu \mathrm{g} / \mathrm{mL})$ were then added to MDAMB-468 cells and 3T3/HER2 cells, respectively. The cells were incubated for either one or eight hours followed by washing once with PBS, and fluorescence microscopy was performed using an Olympus BX81 microscope (Olympus America, Inc., Melville, NY, USA) equipped with the following filters: excitation wavelength 590 to $650 \mathrm{~nm}$ and 672.5 to $747.5 \mathrm{~nm}$, emission wavelength 662.5 to $747.5 \mathrm{~nm}$ and 765 to $855 \mathrm{~nm}$ for Alexa680 and ICG, respectively. Transmitted light differential interference contrast images were also acquired. To validate the specific binding of the antibody, excess antibody $(100 \mu \mathrm{g})$ was used to block $10 \mu \mathrm{g}$ of dye-antibody conjugates.

\section{Flow cytometry studies}

Fluorescence signal from cells after incubation with PanAlexa680 (ON, SQ) or Tra-ICG was measured using a FACS Calibur flow cytometer (BD Biosciences, San Jose, CA, USA) and CellQuest software (BD Biosciences). Briefly, MDA-MB-468 and 3T3/HER2 cells $\left(1 \times 10^{5}\right)$ were incubated with Pan-Alexa680 (ON or SQ) and Tra-ICG at $37^{\circ} \mathrm{C}$ for one or eight hours, respectively. To validate the 
specific binding of the antibody, excess antibody $(50 \mu \mathrm{g})$ was used to block $0.5 \mu \mathrm{g}$ of dye-antibody conjugates.

\section{Tumor model}

All procedures were carried out in compliance with the Guide for the Care and Use of Laboratory Animal Resources (1996), National Research Council, and approved by the National Cancer Institute Animal Care and Use Committee. Six- to eight-week-old female homozygote athymic nude mice were purchased from Charles River (NCI-Frederick, Frederick, MD, USA). During the procedure, mice were anesthetized with isoflurane. MDAMB-468 cells $\left(2 \times 10^{6}\right.$ cells $)$ were injected subcutaneously into the right mammary pads of the mice, and two weeks later (because they grow faster), 3T3/HER2 cells $\left(2 \times 10^{6}\right.$ cells) were injected into the left mammary pads. The experiments were conducted at six days after 3T3/HER2 cell injection.

\section{In vivo two-color two-activatable imaging targeted for EGFR and HER2}

A mixture of Tra-ICG and Pan-Alexa680(SQ or ON) (each $50 \mu \mathrm{g}$ ) was injected via the tail vein into tumorbearing mice (MDA-MB-468 and 3T3/HER2). The mice were anesthetized with intraperitoneally administered $10 \%$ sodium pentobarbital, and fluorescence images were obtained for four days with a flurescence camera (Pearl Imager LI-COR Biosciences using the 700 and $800 \mathrm{~nm}$ fluorescence channel or Maestro in vivo Imaging System CRi) using two filter sets). The red filter sets were used to image Alexa680 fluorescence and the NIR filter sets were used for ICG fluorescence. The red filter set uses a bandpass filter, which ranges between 615 to $665 \mathrm{~nm}$ (excitation) and a long-pass filter over $700 \mathrm{~nm}$ (emission); the NIR filter set uses a band-pass filter from 710 to $760 \mathrm{~nm}$ (excitation) and a long-pass filter over $800 \mathrm{~nm}$ (emission). The tunable emission filter was automatically stepped in $10 \mathrm{~nm}$ increments from 650 to $950 \mathrm{~nm}$ for the red and NIR filter sets at constant exposure. The spectral fluorescence images consist of autofluorescence spectra and the spectra from Alexa680 and ICG, which were then unmixed, based on their spectral patterns using commercial software (Maestro software; CRi). Mice were sacrificed with carbon dioxide immediately after in vivo imaging. The tumors were excised and ex vivo imaging was performed using Pearl Imager and Maestro in vivo imaging system.

\section{Statistical analysis}

Quantitative data were expressed as mean \pm SD. Means were compared using two-way factorial ANOVA followed by the Tukey-Kramer test. $P$-values of $<0.05$ were considered statistically significant.

\section{Results}

Quenching capacity of dye conjugated antibody

The quenching capacities measured by adding 1\% SDS to dye-conjugated antibody were 50-, 5.2- and 1.2-fold for Tra-ICG, Pan-Alexa680(SQ), and Pan-Alexa680 $(\mathrm{ON})$, respectively.

\section{In vitro fluorescent characterization of probes}

In the microscopy studies (Figure 1), the fluorescence signals from Pan-Alexa680(ON) and Pan-Alexa680(SQ) were observed on the surface of MDA-MB-468 cells one hour after incubation. Both probes also showed fluorescent signal within the cells eight hours after incubation, and the fluorescent dots were brighter for Pan-Alexa680 (SQ) than for Pan-Alexa680(ON). These signals were completely blocked by the addition of excess panitumumab. Similarly, fluorescence signal from Tra-ICG was observed within the 3T3/HER2 cells. While signal was minimal at one hour after incubation because of greater quenching magnitude of Tra-ICG than Pan-Alexa680, many bright intracellular foci were present by eight hours, and these signals were blocked by excess trastuzumab.

FACS studies were similar to microscopy studies, that is, Pan-Alexa680(ON, SQ) and Tra-ICG exhibited specific binding to MDA-MB-468 and 3T3/HER2 cells, respectively (Figure 2). Pan-Alexa680(SQ) showed progressively brighter signal over time compared with Pan-Alexa680 $(\mathrm{ON})$ where the signal was lower and constant.

\section{In vivo two-color activatable imaging targeted for EGFR and HER2}

Figure 3A shows the images of tumor-bearing mice (MDA-MB-468 and 3T3/HER2) injected with the mixture of Pan-Alexa680(ON or SQ) and Tra-ICG, which was obtained with the Pearl Imager. Pan-Alexa680(ON) detected the targeted tumor (MDA-MB-468, EGFR+); however, high background and non-specific tumor uptake (3T3/HER2) are evident on Day 1 through Day 3 post injection. At Day 4, relatively selective images of EGFR positive (MDA-MB-468) tumors were obtained. In contrast, Pan-Alexa680(SQ) clearly visualized MDA-MB468 with high tumor-to-background ratios as early as Day 2 and at all later time points, although a slight signal was detected in the 3T3/HER2 tumor and bladder at Day 1. On the other hand, Tra-ICG specifically visualized the HER2 positive 3T3/HER2 tumors, although slight fluorescent signals were noticed in the liver and intestine. Tumor-to-background (non-targeted tumors, liver, and neck) ratios were significantly higher $(P<0.01)$ for PanAlexa680(SQ) compared with Pan-Alexa680(ON) for four days (Figure 3B). Tumor-to-non-targeted tumor, tumor-liver and tumor-neck ratios were 8.1, 10.3, and 8.8 


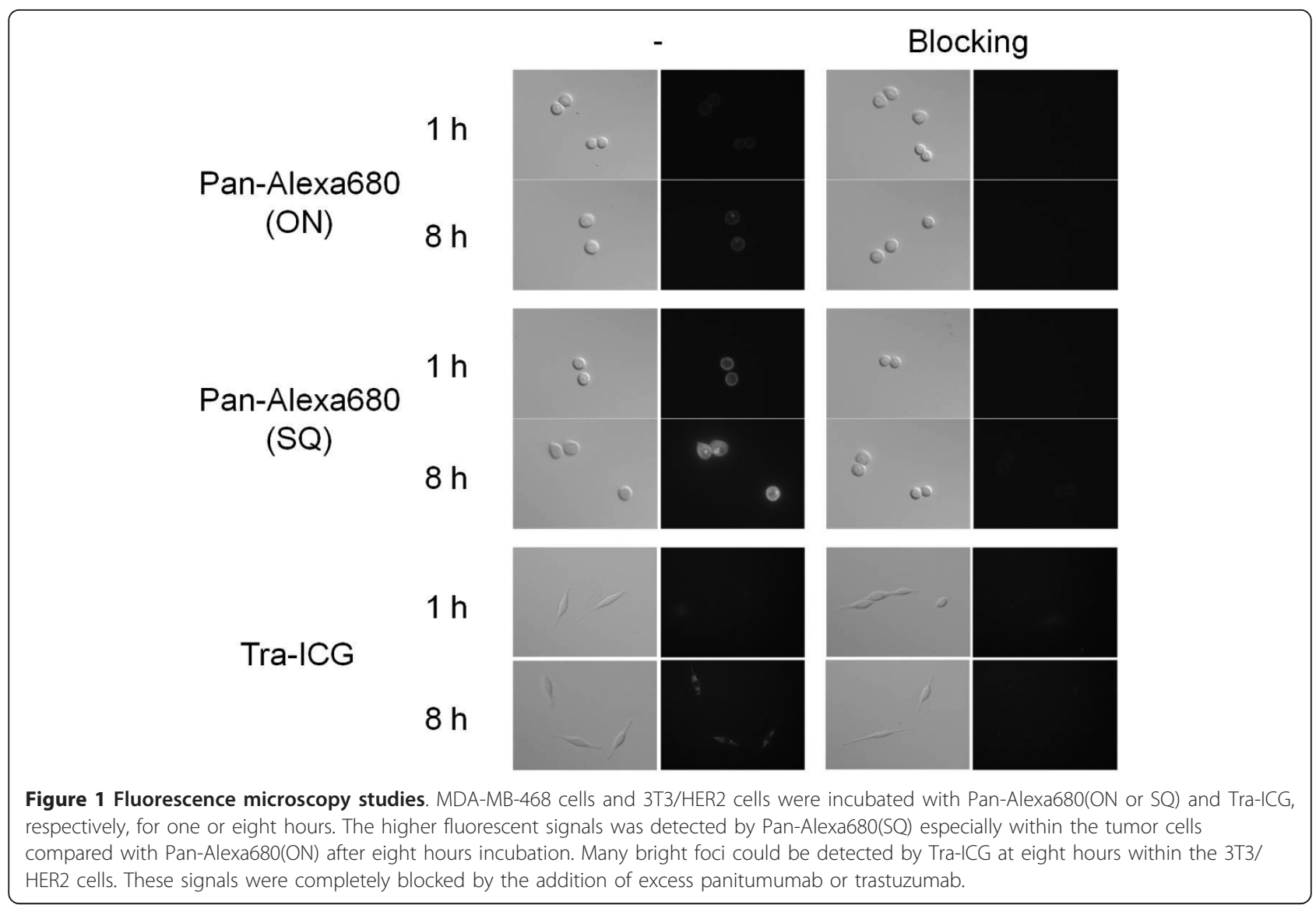

for Pan-Alexa680(SQ) at Day 4; whereas, they were 2.7, 3.9, and 4.5 for Pan-Alexa680(ON). In contrast, tumorto-background ratios with Tra-ICG were not significantly different between the two groups (Figure 3C). The Maestro spectral fluorescence imager provided similar images to the Pearl imager (Figure 4). For instance, tumor-tonon-targeted tumor, tumor-liver and tumor-neck were 8.1, 6.7, and 7.9 for Pan-Alexa680(SQ) at Day 4, whereas, these same ratios were 2.8, 3.0, and 4.2 for Pan-Alexa680 (ON). Tumor-to-background ratios on Tra-ICG were not significantly different between both groups.

\section{Discussion}

Activatable optical imaging using a labeled targeting antibody can be a sensitive and specific method of performing in vivo molecular imaging with high tumor-to-background ratios [6]. Like immunohistochemistry, each antibody can be labeled with a different type of activatable fluorophore allowing multi-color imaging. In this case, two different EGFR receptors (EGFR and HER2) were targeted successfully.

Activatable fluorescence probes can be based on one of several mechanisms, including self-quenching (Homo-FRET), auto-quenching (Hetero-FRET), H-dimer formation, photo-induced electron transfer (PeT) and uncaging [6]. Among these, self-quenching and autoquenching fluorophores are relatively easy to synthesize. Both antibody conjugates showed intense bright fluorescence foci within tumor cells expressing the respective targets, suggesting internalization of the antibody conjugate was required for activation $[7,10]$.

In the case of the two activatable antibody conjugates described here, Tra-ICG and Pan-Alexa680(SQ), we showed that they could achieve impressive dequenching ratios of 50- and 5.2-fold respectively. The difference of dequenching capacity for ICG and Alexa680 can be explained by distinct quenching mechanisms of two conjugates. The fluorescence of ICG can be quenched by autoquenching that is hetero-FRET between ICG and aromatic amino acids of antibodies; in contrast, the fluorescence of Alexa680 can be quenched by homo-FRET between two or more Alexa680 molecules in a conjugate. Therefore, greater numbers of Alexa680 on a single antibody could achieve better quenching of Pan-Alexa680(SQ). However, the bio-distribution was dramatically changed and showed high uptake in the liver, when seven or more Alexa680s were conjugated with a single panitumumab. Thus, the number of Alexa680 molecules conjugated with a single 

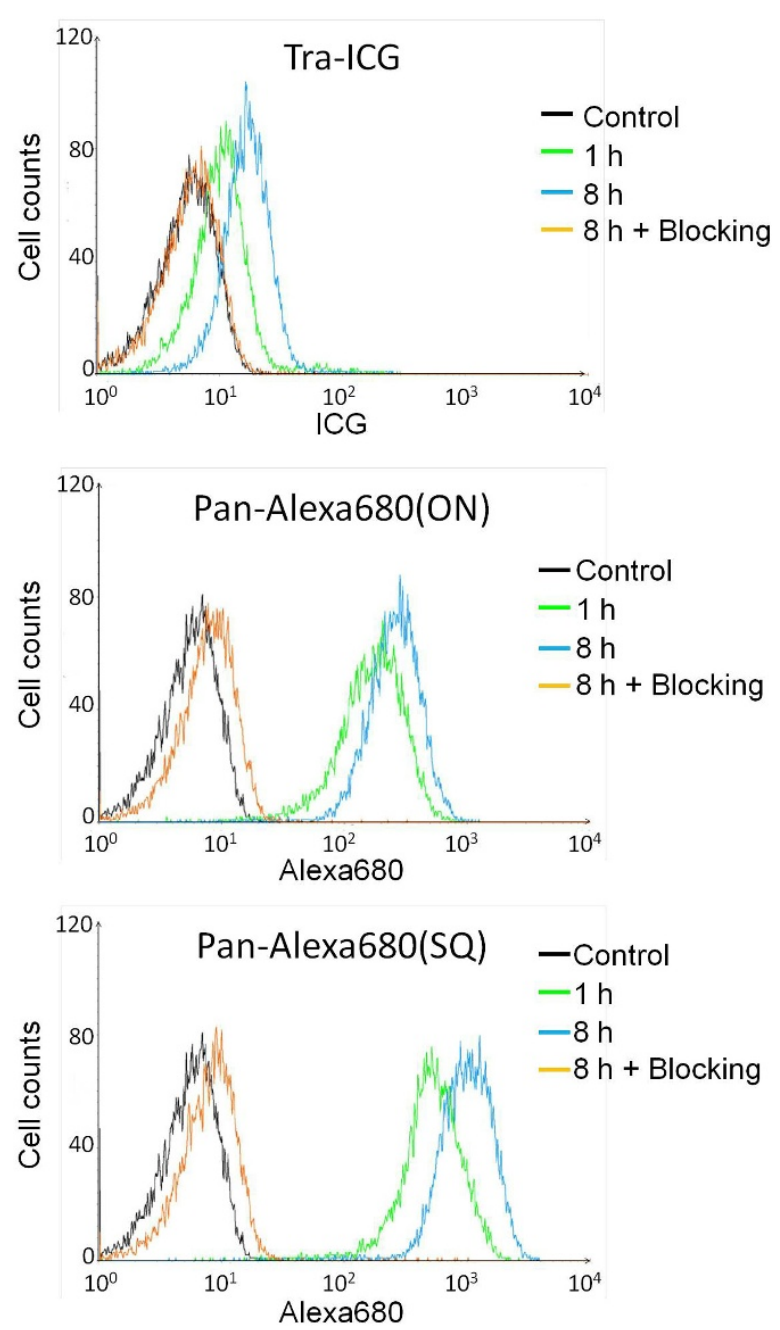

Figure 2 Flow cytometry studies. Flow cytometry studies showed specific binding of Pan-Alexa680(ON or SQ) and Tra-ICG to EGFR and HER2, respectively. Pan-Alexa680(SQ) showed brighter signals and increased fluorescent signals over time compared with Pan-Alexa680(ON).

antibody had to be limited up to five despite of incomplete self-quenching of Pan-Alexa(SQ). Therefore, fluorescence signal of activated Pan-Alexa680(SQ) was greater; however, the dequenching ratios of Pan-Alexa(SQ) were lower than that of Tra-ICG.

We employed two imaging devices in this study. The Pearl imager (Pearl, LI-COR Biosciences) can detect two kinds of NIR probes (emission wavelength 700 and 800 $\mathrm{nm}$ ) separately by using optical filter sets and a highly sensitive CCD camera. However, the multispectral fluorescence imager (Maestro, CRi) is equipped with a tunable crystal filter that can distinguish between two or more distinct fluorescence signals emanating from different external and internal fluorophores by their spectral separation [11]. The former system can simply show photon counts at the specific range of wavelength, while the latter system can extract the spectral signal from each distinct fluorophores. As shown in the results, spectral unmixing is able to separate the different colors than optical filtering, allowing multi-targeted color images with high tumor-to-background ratios.

In contrast to previous studies, which employed subcutaneous xenografts, we employed orthotopic bilateral breast cancer tumor models, each one of which expressed either EGFR or HER2. This enabled us to use the orthotopic tumors as controls for each other [12]. A potential alternative will be the use of fluorescent proteins, which are excellent endogenous fluorescence emitters to be used as a powerful tool for depicting various biological processes both in vitro and in vivo [13]. However, for the medical application, fluorescence proteins require in vivo virus-mediated gene transfection $[14,15]$, which is unlikely to be permitted for the diagnosis in humans at least in the near term.

Since tumors show genomic and proteomic diversity, expression profiles vary widely even within a single 


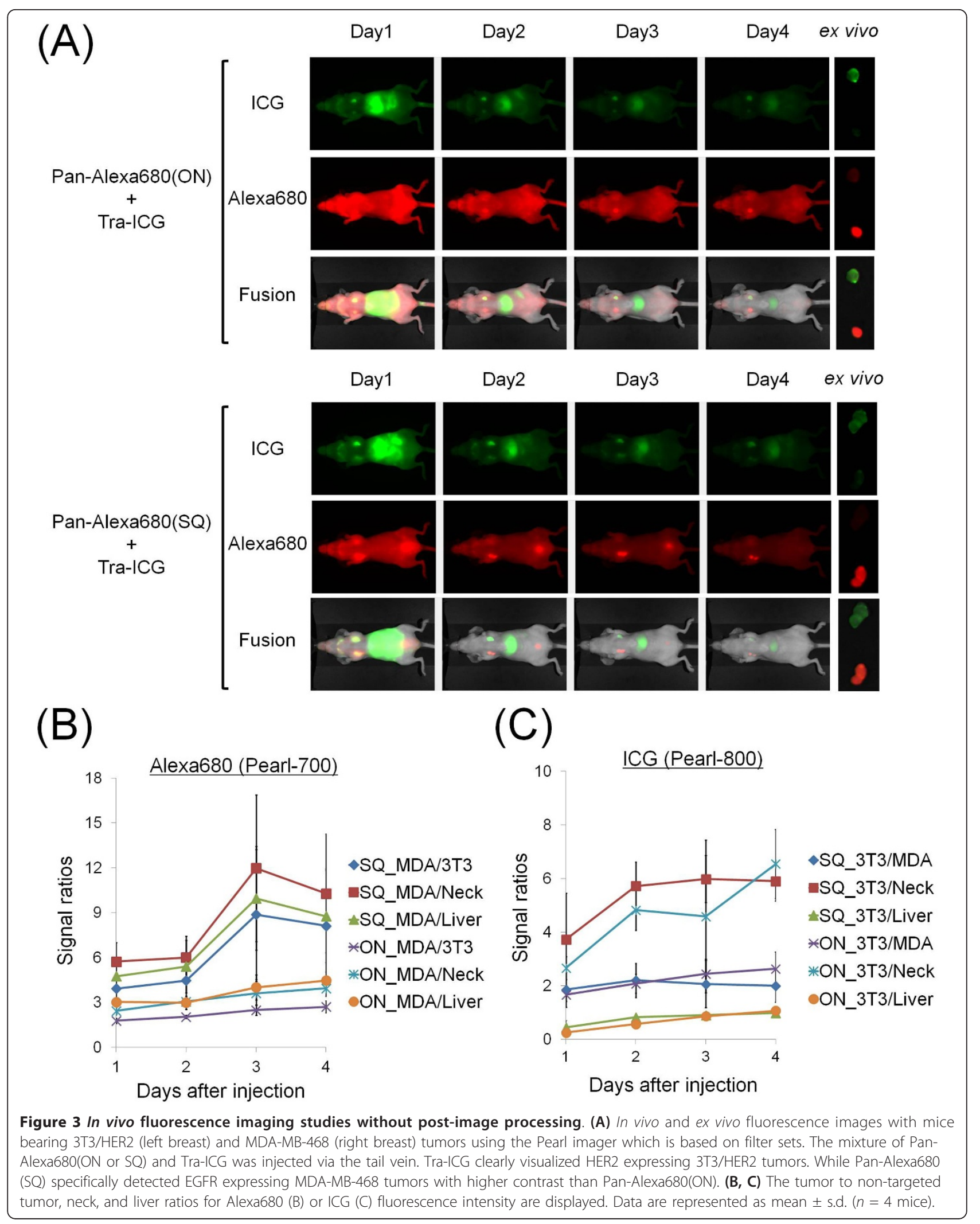




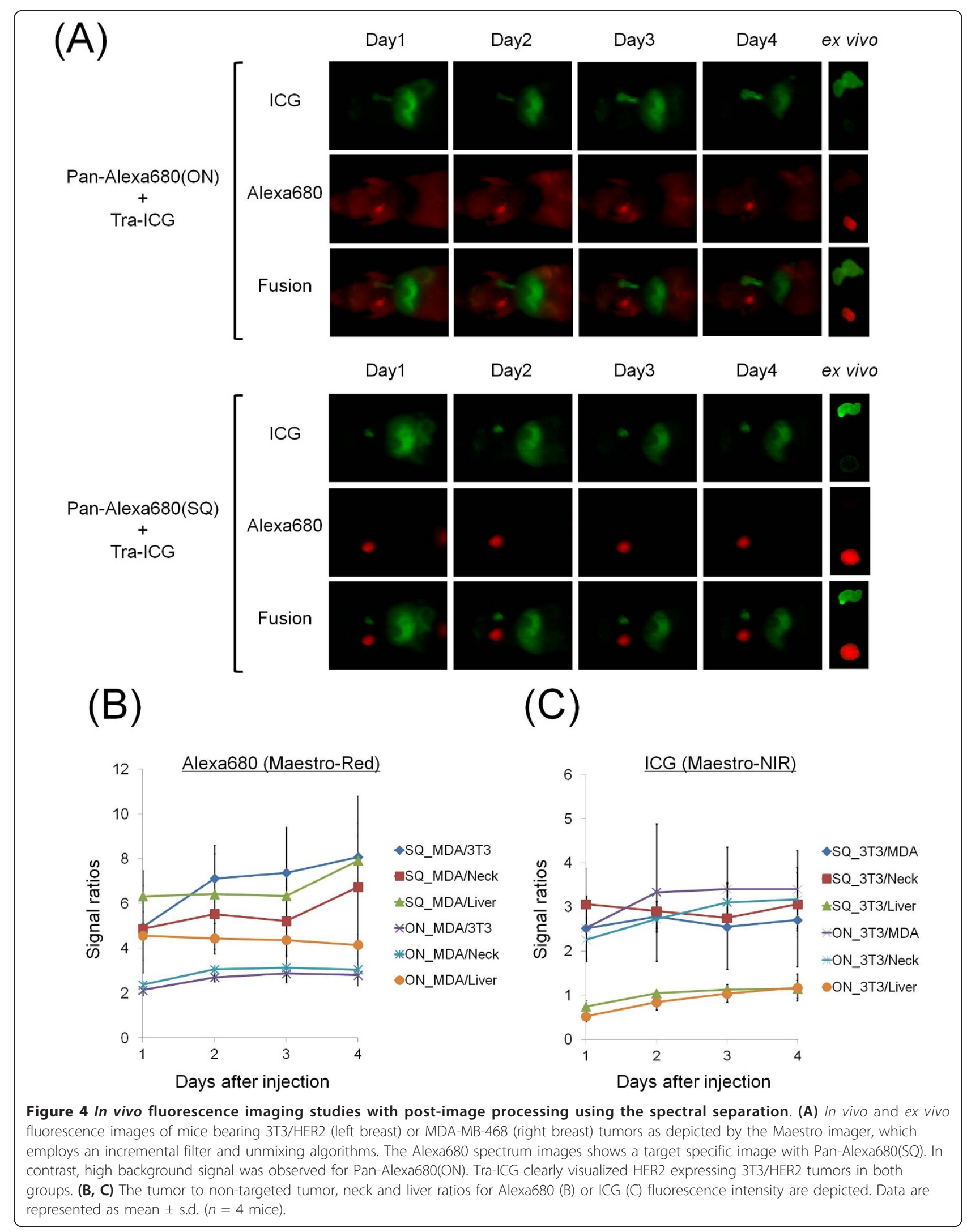


lesion. EGFR and HER2 play an important role in carcinogenesis; overexpression of one or more members of the EGFR family has been shown in a number of malignancies, including breast, ovarian, non-small cell lung cancer and squamous cell carcinomas. Furthermore, high levels of expression relates to a poor prognosis [16-18]. However, currently it is necessary to extract tissue and stain using immunohistochemistry methods. Detecting expression levels of EGFR profile in vivo could be helpful for detecting and characterizing malignant tumors, as well as determining therapy.

\section{Conclusion}

By employing two-color, activatable fluorophores conjugated to specific antibodies, multi-target specific fluorescence imaging could be achieved with high target-tobackground ratios that allowed us to see a specific receptor expression in each breast tumor without postprocessing images. Such methods may be useful in the future for in vivo characterization of breast cancers.

\section{Abbreviations \\ EGFR: epidermal growth factor receptor; HER1: human epidermal growth factor receptor type 1; HER2: human epidermal growth factor receptor type 2; ICG: Indocyanine Green; IHC: immunohistochemistry; mAb: monoclonal antibody; NIR: near infrared; ON: always on; Pan: panitumumab; PBS: phosphate-buffered saline; SDS: sodium dodecyl sulfate; SQ: self-quenched; Tra: trastuzumab.}

\section{Acknowledgements}

This research was supported by the Intramural Research Program of the US National Institutes of Health, National Cancer Institute, Center for Cancer Research.

\section{Authors' contributions}

KS conducted experiments, performed analysis and wrote the manuscript. MM and TN conducted experiments and performed analysis. PLC wrote the manuscript and supervised the project, while HK planned and initiated the project, designed and conducted experiments, wrote the manuscript, and supervised the entire project. All authors read and approved the final manuscript.

\section{Competing interests}

The authors declare that they have no competing interests.

Received: 19 February 2012 Revised: 14 March 2012

Accepted: 17 April 2012 Published: 17 April 2012

\section{References}

1. Wu AM, Senter PD: Arming antibodies: prospects and challenges for immunoconjugates. Nat Biotechnol 2005, 23:1137-1146.

2. Kobayashi H, Longmire MR, Ogawa M, Choyke PL: Rational chemical design of the next generation of molecular imaging probes based on physics and biology: mixing modalities, colors and signals. Chem Soc Rev 2011, 40:4626-4648.

3. Kobayashi H, Longmire MR, Ogawa M, Choyke PL, Kawamoto S: Multiplexed imaging in cancer diagnosis: applications and future advances. Lancet Oncol 2010, 11:589-595.

4. Barrett $T$, Koyama $Y$, Hama $Y$, Ravizzini $G$, Shin IS, Jang BS, Paik CH, Urano $Y$ Choyke $\mathrm{PL}$, Kobayashi $\mathrm{H}$ : In vivo diagnosis of epidermal growth factor receptor expression using molecular imaging with a cocktail of optically labeled monoclonal antibodies. Clin Cancer Res 2007, 13:6639-6648.
5. Koyama Y, Barrett T, Hama Y, Ravizzini G, Choyke PL, Kobayashi H: In vivo molecular imaging to diagnose and subtype tumors through receptortargeted optically labeled monoclonal antibodies. Neoplasia 2007, 9:1021-1029.

6. Kobayashi H, Choyke PL: Target-cancer-cell-specific activatable fluorescence imaging probes: rational design and in vivo applications. Acc Chem Res 2011, 44:83-90

7. Ogawa M, Kosaka N, Choyke PL, Kobayashi $\mathrm{H}$ : In vivo molecular imaging of cancer with a quenching near-infrared fluorescent probe using conjugates of monoclonal antibodies and indocyanine green. Cancer Res 2009, 69:1268-1272.

8. Kobayashi H, Ogawa M, Alford R, Choyke PL, Urano Y: New strategies for fluorescent probe design in medical diagnostic imaging. Chem Rev 2010, 110:2620-2640.

9. Ogawa M, Kosaka N, Longmire MR, Urano Y, Choyke PL, Kobayashi H: Fluorophore-quencher based activatable targeted optical probes for detecting in vivo cancer metastases. Mol Pharm 2009, 6:386-395.

10. Ogawa M, Regino CA, Choyke PL, Kobayashi H: In vivo target-specific activatable near-infrared optical labeling of humanized monoclonal antibodies. Mol Cancer Ther 2009, 8:232-239.

11. Maihle NJ, Baron AT, Barrette BA, Boardman CH, Christensen TA, Cora EM, Faupel-Badger JM, Greenwood T, Juneja SC, Lafky JM, Lee H, Reiter JL, Podratz KC: EGF/ErbB receptor family in ovarian cancer. Cancer Treat Res 2002, 107:247-258

12. Hoffman RM: Orthotopic metastatic mouse models for anticancer drug discovery and evaluation: a bridge to the clinic. Invest New Drugs 1999, 17:343-359.

13. Kishimoto H, Aki R, Urata Y, Bouvet M, Momiyama M, Tanaka N, Fujiwara T, Hoffman RM: Tumor-selective, adenoviral-mediated GFP genetic labeling of human cancer in the live mouse reports future recurrence after resection. Cell Cycle 2011, 10:2737-2741.

14. Kishimoto H, Urata $Y$, Tanaka N, Fujiwara T, Hoffman RM: Selective metastatic tumor labeling with green fluorescent protein and killing by systemic administration of telomerase-dependent adenoviruses. $\mathrm{Mol}$ Cancer Ther 2009, 8:3001-3008.

15. Kishimoto $H$, Zhao M, Hayashi $K$, Urata $Y$, Tanaka N, Fujiwara T, Penman S, Hoffman RM: In vivo internal tumor illumination by telomerasedependent adenoviral GFP for precise surgical navigation. Proc Natl Acad Sci USA 2009, 106:14514-14517.

16. Gullick WJ, Marsden JJ, Whittle N, Ward B, Bobrow L, Waterfield MD: Expression of epidermal growth factor receptors on human cervical, ovarian, and vulval carcinomas. Cancer Res 1986, 46:285-292.

17. Salomon DS, Brandt R, Ciardiello F, Normanno N: Epidermal growth factorrelated peptides and their receptors in human malignancies. Crit Rev Oncol Hematol 1995, 19:183-232.

18. Souder C, Leitzel K, Ali SM, Demers L, Evans DB, Chaudri-Ross HA, Hackl W, Hamer P, Carney W, Lipton A: Serum epidermal growth factor receptor/ HER-2 predicts poor survival in patients with metastatic breast cancer. Cancer 2006, 107:2337-2345.

doi: $10.1186 /$ bcr3167

Cite this article as: Sano et al:: In vivo breast cancer characterization imaging using two monoclonal antibodies activatably labeled with near infrared fluorophores. Breast Cancer Research 2012 14:R61.

\section{Submit your next manuscript to BioMed Central and take full advantage of:}

- Convenient online submission

- Thorough peer review

- No space constraints or color figure charges

- Immediate publication on acceptance

- Inclusion in PubMed, CAS, Scopus and Google Scholar

- Research which is freely available for redistribution 